\title{
A New Method of Resolution of the Bending of Thick FGM Beams Based on Refined Higher Order Shear Deformation Theory
}

\author{
Abderrahim Razouki*, Lhoucine Boutahar, Khalid El Bikri \\ Mohammed V University of Rabat, ENSET- Rabat, M2SM, B.P.6207, Rabat Instituts 10100, Rabat, Morocco
}

Received January 19, 2020; Revised February 26, 2020; Accepted March 12, 2020

Copyright $\odot 2020$ by authors, all rights reserved. Authors agree that this article remains permanently open access under the terms of the Creative Commons Attribution License 4.0 International License

\begin{abstract}
The aim of this work is to study the static bending of functionally graded beams accounting higher order of shear deformation theory. The governing equations, derived from the virtual work principle, are a set of ordinary differential equations describing a static bending of a thick beam. Thus, this paper presents the differential transform method used to solve the previous system of equations. The results obtained lay the foundation to determine the exact analytical solution for different boundary conditions and external loadings. The axial displacement and the bending and shear displacements, in the exact analytical form, of a thick clamped-clamped beam with functionally graded material under a uniform load will be fully developed. Moreover, normal and shear stresses will be analyzed. To confirm the efficiency of this work, a comparis on with the numerical results provided by literature is performed. Through this work, the given analytical results provide engineers with an accurate tool to determine the analytical solution for the bending of plates and shells. In addition, the geometric and material parameters that appear clearly in the analytical results allow for a more optimized design of functionally graded material beams. This type of beams is frequently used in mechanical engineering fields such as aerospace engineering.
\end{abstract}

Keywords Differential Transformation Method, Exact Solution, Static Analysis, Clamped-Clamped Beam, Bending, Functionally Graded Beam

\section{Introduction}

The bending of thick functionally graded (FG) beams continues to occupy an important spot in mechanical engineering. Therefore, it is necessary to provide engineers with analytical results, enabling them to predict the bending behavior of FG thick beamsin a parametric way and thus improve the performance of systems in a competitive manner.

Using a finite element method, R. Kadoli et al [1] studied the static bending behavior of FG beams with higher order shear deformation beam theory (HOSDT). Simsek [2] developed a numerical solution for Timoshenko beam theory (TBT) and the HOSDT using Ritz method. Giunta Gand al [3] adopted Navier closed form solution to solve classical beam theories. Zhong [4] and Li et al [5] presented analytical solution for cantilever FG beams. Vo, Thuc and al [6] presented finite element solution with Hermite interpolation based on refined shear deformation theory. Farhatnia and al (2019) [18] developed a finite ele mentary approach to study the bending and buckling FG beam based on refined Zigzag Theory. Razouki and al (2019) [19] applied differential transform method(DTM) and gave the exact analytical solution to the bending FG beam based on higher order shear deformation theory (simply supported - simply supported beam case).

In this paper, DTM is applied to the governing equations, which are obtained from the principle of virtual work, to give a general solution [19]. The general exact solution form for a bending FG beam with various higher-order shear deformation beam theories is fully developed. The exact solution is given for clamped-clamped beam $(\mathrm{C}-\mathrm{C})$ subjected to a uniformly distributed load (UDL). The results are compared with the existing numerical ones to validate the obtained solution. In addition, the analytical expression for transverse deflection is given to show the effect of shear displacement.

\section{Constitutive Relations}

Consider a FG beam with length $\mathrm{L}$ and rectangular 
cross-section $\mathrm{b} x \mathrm{~h}$, with $\mathrm{b}$ being the width and $\mathrm{h}$ being the height as shown in fig 1.
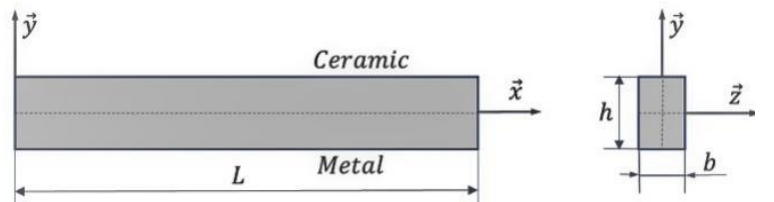

Figure 1. FG beam - Geometry and coordinates

FGMs are composite materials made of ceramic and metal. The material's properties of FG beams are assumed to vary continuously through the depth of the beam according to a power law as $[1,2,5,13]$

$$
P(y)=\left(P_{c}-P_{m}\right)\left(\frac{y}{h}+\frac{1}{2}\right)^{n}+P_{m}
$$

\section{Theory and Formulation}

\subsection{Displacement Fields and Strains}

The displacement fields of various higher-order shear deformation beam theories are given in a general form as $[6,14,15,17]$.

$$
\begin{gathered}
u_{1}(x, y)=u(x)-y \frac{d w_{b}}{d x}-f(y) \frac{d w_{s}}{d x} \\
u_{2}(x, y)=w_{b}(x)+w_{s}(x) \\
u_{3}(x, y)=0
\end{gathered}
$$

where $\mathrm{u}$ is the axial displacement of a point on the midplane of the beam; $w_{b}$ and $w_{s}$ are the bending and shear components of trans verse dis placement of a point on the midplane of the beam. $f(y)$ is a shape function indicating the distribution of the trans verse shear strain and shear stress through the depth of the beam [6].

The non-zero strains are given by:

The axial strain

$$
\varepsilon_{x}=\frac{d u}{d x}-y \frac{d^{2} w_{b}}{d x^{2}}-f(y) \frac{d^{2} w_{s}}{d x^{2}}
$$

The shear strain

$$
\gamma_{x y}=g(y) \frac{d w_{s}}{d x} ; g(y)=\left(1-\frac{d f(y)}{d y}\right)
$$

By assuming that the material of FG beam obeys Hooke's law, the stresses in the beam become

$$
\begin{gathered}
\sigma_{\mathrm{x}}=\mathrm{E}(\mathrm{y}) \varepsilon_{\mathrm{x}} \text { Axial normal stress } \\
\tau_{\mathrm{xy}}=\mathrm{G}(\mathrm{y}) \gamma_{\mathrm{xy}} \text { Shear stress }
\end{gathered}
$$

where G(y) is the Shear modulus related to the Young's modulus E(y) by:

$$
G(y)=\frac{E(y)}{2(1+v(y))}
$$

\subsection{Strain Energy and External Load Work}

The virtual of the strain energy $\mathcal{U}$ of the FG beam is given by $[6,16]$ :

$$
\delta \mathcal{U}=\int\left(\sigma_{\mathrm{x}} \delta \varepsilon_{\mathrm{x}}+\tau_{\mathrm{xy}} \delta \gamma_{x y}\right) \mathrm{d} s \mathrm{dx}
$$

where $\delta$ is the variational symbol, $\mathrm{S}$ the cross-sectional area of the uniform beam.

By giving the virtual forms $\delta \varepsilon_{11}$ and $\delta \gamma_{12}$ from Eq. (3) and substituting the subsequent results into $\mathrm{Eq}$. (5) we obtain $[6,19]$ :

$\delta \mathcal{U}=\int_{0}^{\mathrm{L}}\left(\mathrm{N} \frac{\mathrm{d} \delta \mathrm{u}}{\mathrm{dx}}+\mathrm{M}_{\mathrm{b}} \frac{\mathrm{d}^{2} \delta \mathrm{w}_{\mathrm{b}}}{\mathrm{dx}^{2}}+\mathrm{M}_{\mathrm{s}} \frac{\mathrm{d}^{2} \delta \mathrm{w}_{\mathrm{s}}}{\mathrm{dx}^{2}}+\mathrm{Q} \frac{\mathrm{d} \delta \mathrm{w}_{\mathrm{s}}}{\mathrm{dx}}\right) \mathrm{dx}(6)$

Where $N, M_{b}, M_{s}$ and $Q$ are the stress resultants, defined as:

$$
\begin{aligned}
\left(N, M_{b}, M_{s}\right) & =\int \sigma_{11}(1,-y,-f(y)) d s \\
Q & =\int \tau_{x y} g(y) d s
\end{aligned}
$$

The virtual potential energy of the applied transverse load $\mathrm{q}(\mathrm{x})$ is given by $[6,19]$ :

$$
\delta \mathcal{V}=-\int_{0}^{\mathrm{L}} \mathrm{q}(\mathrm{x})\left(\delta \mathrm{w}_{\mathrm{b}}(\mathrm{x})+\delta \mathrm{w}_{\mathrm{s}}(\mathrm{x})\right) \mathrm{dx}
$$

\subsection{Equilibrium Equations}

The principle of virtual work states that if a body is in equilibrium then the total virtual work done is zero $[15$, $16]$.

$$
\delta \mathcal{U}+\delta \mathcal{V}=0
$$

Substituting the expressions of $\delta \mathcal{U}$ and $\delta \mathcal{V}$ from Eq. (6) and Eq. (8) into Eq. (9) and integrating by parts space variables, and collecting the coefficients of $\delta \mathrm{u}, \delta \mathrm{w}_{\mathrm{b}}$, and $\delta w_{s}$, the following equations of equilibrium of the functionally graded beam are obtained $[6,19]$ :

$$
\begin{gathered}
\delta \mathrm{u}: \frac{\mathrm{dN}}{\mathrm{dx}}=0 \\
\delta \mathrm{w}_{\mathrm{b}}: \frac{\mathrm{d}^{2} \mathrm{M}_{\mathrm{b}}}{\mathrm{dx}^{2}}-\mathrm{q}(\mathrm{x})=0 \\
\delta \mathrm{w}_{\mathrm{s}}: \frac{\mathrm{d}^{2} \mathrm{M}_{\mathrm{s}}}{\mathrm{dx}^{2}}-\frac{\mathrm{dQ}}{\mathrm{dx}}-\mathrm{q}(\mathrm{x})=0
\end{gathered}
$$

The boundary conditions are of the form: specify

$$
\begin{array}{r}
\text { u or } \mathrm{N} ; \mathrm{w}_{\mathrm{b}} \text { or } \frac{d w_{b}}{d x} ; w_{s} \text { or } \frac{d w_{s}}{d x} \text {; } \\
M_{b} \text { or } \frac{d M_{b}}{d x} ; M_{s} \text { or } \frac{d M_{s}}{d x}
\end{array}
$$

By substituting Eqs. (3) and (4) into Eqs. (7), the constitutive equations for the stress resultants are obtained as follows [6,19]:

$$
\begin{gathered}
N=A \frac{d u}{d x}-B \frac{d^{2} w_{b}}{d x^{2}}-B_{s} \frac{d^{2} w_{s}}{d x^{2}} \\
M_{b}=-B \frac{d u}{d x}+D \frac{d^{2} w_{b}}{d x^{2}}+D_{s} \frac{d^{2} w_{s}}{d x^{2}} \\
M_{s}=-B_{s} \frac{d u}{d x}+D_{s} \frac{d^{2} w_{b}}{d x^{2}}+H_{s} \frac{d^{2} w_{s}}{d x^{2}}
\end{gathered}
$$




$$
Q=A_{s} \frac{d w_{s}}{d x}
$$

Where

$$
\begin{gathered}
(A, B, D)=\int\left(1, y, y^{2}\right) E(y) d s \\
\left(B_{s}, D_{s}, H_{s}\right)=\int\left(f(y), y f(y), f(y)^{2}\right) E(y) d s \\
A_{s}=\int g(y)^{2} G(y) d s
\end{gathered}
$$

The coefficients of the Eqs. (13) are given in the appendix A.

Then, Eqs (10) can be expressed in terms of the displacements $\mathrm{u}, \mathrm{w}_{\mathrm{b}}$ and $\mathrm{w}_{\mathrm{s}}$ as follows [18]:

$$
\begin{gathered}
\delta u: A \frac{d^{2} u}{d x^{2}}-B \frac{d^{3} w_{b}}{d x^{3}}-B_{s} \frac{d^{3} w_{s}}{d x^{3}}=0 \\
\delta w_{b}:-B \frac{d^{3} u}{d x^{3}}+D \frac{d^{4} w_{b}}{d x^{4}}+D_{s} \frac{d^{4} w_{s}}{d x^{4}}-q(x)=0 \\
\delta w_{s}:-B_{s} \frac{d^{3} u}{d x^{3}}+D_{s} \frac{d^{4} w_{b}}{d x^{4}}+H_{s} \frac{d^{4} w_{s}}{d x^{4}}-A_{s} \frac{d^{2} w_{s}}{d x^{2}}-q(x)= \\
0
\end{gathered}
$$

\section{Differential Transform Method}

The differential transformation is defined as follows $[7,8,9,10,11,12]$ :

$$
F(k)=F_{k}=\frac{1}{k !}\left[\frac{d^{k} f(x)}{d x^{k}}\right]_{x_{0}}
$$

in which $f(x)$ the original function, $F(k)=F_{k}$ is the transformed function. The inverse differential transformation is defined as:

$f(x)=\sum_{k=0}^{\infty} F_{k}\left(x-x_{0}\right)^{k}=\sum_{k=0}^{\infty}\left(\frac{1}{k !}\left[\frac{d^{k_{f}}(x)}{d^{k} x_{x_{0}}}\left(x-x_{0}\right)^{k}\right)\right.$

Table 1 gives the main operations performed by differential transformation.

Table 1. One-variable differential transform functions

\begin{tabular}{|l|l|}
\hline Original function & Transformedfunction \\
\hline$f(x)=a \cdot u(x) \pm b \cdot v(x)$ & $F_{k}=a \cdot U_{k} \pm b \cdot V_{k}$ \\
\hline$f(x)=x^{m}$ & $F_{k}=\delta(k-m)=\left\{\begin{array}{l}1, k=m \\
0, k \neq m\end{array}\right.$ \\
\hline$f(x)=\frac{d^{m} u(x)}{d x^{m}}$ & $F_{k}=\frac{(k+m) !}{k !} U_{k+m}$ \\
\hline$f(x)=e^{a x}$ & $F_{k}=\frac{a^{k}}{k !}$ \\
\hline$f(x)=\sin (\omega x+\alpha)$ & $F_{k}=\frac{\omega^{k}}{k !} \sin \left(\frac{\pi k}{2}+\alpha\right)$ \\
\hline$f(x)=\cos (\omega x+\alpha)$ & $F_{k}=\frac{\omega^{k}}{k !} \cos \left(\frac{\pi k}{2}+\alpha\right)$ \\
\hline$f(x)=u(x) v(x)$ & $F_{k}=\sum_{l=0}^{k} U_{1} V_{k-1}$ \\
\hline
\end{tabular}

\section{General Form of the Exact Analytical Solution}

\subsection{DTM and the Equilibrium System}

Assuming that the solutions of the shear $\mathrm{w}_{\mathrm{s}}(\mathrm{x})$, bending $\mathrm{w}_{\mathrm{b}}(\mathrm{x})$, axial $\mathrm{u}(\mathrm{x})$ displacements and external load $\mathrm{q}(\mathrm{x})$ are a polynomial forms (power series) as follows [19]:

$$
\begin{array}{r}
\mathrm{w}_{\mathrm{s}}(\mathrm{x})=\sum_{\mathrm{k}=0}^{\infty} \mathrm{s}_{\mathrm{k}} \mathrm{x}^{\mathrm{k}} \\
\mathrm{w}_{\mathrm{b}}(\mathrm{x})=\sum_{\mathrm{k}=0}^{\infty} \mathrm{b}_{\mathrm{k}} \mathrm{x}^{\mathrm{k}} \\
\mathrm{u}(\mathrm{x})=\sum_{\mathrm{k}=0}^{\infty} \mathrm{u}_{\mathrm{k}} \mathrm{x}^{\mathrm{k}} \\
\mathrm{q}(\mathrm{x})=\sum_{\mathrm{k}=0}^{\infty} \mathrm{q}_{\mathrm{k}} \mathrm{x}^{\mathrm{k}}
\end{array}
$$

And applying the DTM (for $\mathrm{x}_{0}=0$ ) to Eqs. (17) and Eqs. (14) leads to a recurrent system as follows:

For $\mathrm{k}=0,1,2,3, \ldots$

$$
\begin{aligned}
& \mathrm{u}_{\mathrm{k}+2}=\mathrm{A}_{1} \lambda_{k+3}^{-1} \mathrm{~b}_{\mathrm{k}+3}+\mathrm{A}_{2} \lambda_{k+3}^{-1} \mathrm{~s}_{\mathrm{k}+3} \\
\mathrm{~b}_{\mathrm{k}+4}= & \mathrm{B}_{1} \lambda_{k+3}^{1} \mathrm{u}_{\mathrm{k}+3}+\mathrm{B}_{2} \lambda_{k+2}^{2} \mathrm{~s}_{\mathrm{k}+2}+\mathrm{B}_{3} \lambda_{k}^{4} \mathrm{q}_{\mathrm{k}} \\
\mathrm{s}_{\mathrm{k}+4}= & \mathrm{C}_{1} \lambda_{k+3}^{1} \mathrm{u}_{\mathrm{k}+3}+\mathrm{C}_{2} \lambda_{k+2}^{2} \mathrm{~s}_{\mathrm{k}+2}+\mathrm{C}_{3} \lambda_{k}^{4} \mathrm{q}_{\mathrm{k}}
\end{aligned}
$$

In which

$$
\lambda_{m}^{a}=\frac{m !}{(m+a) !}
$$

And where $A_{1}, A_{2}, B_{1}, B_{2}, B_{3}, C_{1}, C_{2}$ and $C_{3}$ are given by the appendix $B$

\subsection{Displacements Sequences}

\subsubsection{Axial Displacement Sequence}

By substituting Eq. (18b) and Eq.(18c) into Eq. (18a) we obtain the following recurrent axial displacement sequence:

$$
\mathrm{u}_{2}=3 \mathrm{~A}_{1} \mathrm{~b}_{3}+3 \mathrm{~A}_{2} \mathrm{~s}_{3}
$$

For $\mathrm{k}=1,2,3, \ldots$

$$
\mathrm{u}_{\mathrm{k}+2}=\lambda_{\mathrm{k}+1}^{1} \mathrm{k}_{\mathrm{u}} \mathrm{s}_{\mathrm{k}+1}+\lambda_{\mathrm{k}-1}^{3} \mathrm{E}_{\mathrm{u}} \mathrm{q}_{\mathrm{k}-1}
$$

where

$$
\mathrm{k}_{\mathrm{u}}=\frac{\Delta^{* \phi}}{\Delta_{0}}, \mathrm{E}_{\mathrm{u}}=\frac{\Delta^{* \mathrm{E}}}{\Delta_{0}}
$$

And

$$
\begin{gathered}
\Delta_{0}=1-\mathrm{A}_{1} \mathrm{~B}_{1}-\mathrm{A}_{2} \mathrm{C}_{1} \\
\Delta^{* \phi}=\mathrm{A}_{1} \mathrm{~B}_{2}+\mathrm{A}_{2} \mathrm{C}_{2} \\
\Delta^{* \mathrm{E}}=\mathrm{A}_{1} \mathrm{~B}_{3}+\mathrm{A}_{2} \mathrm{C}_{3}
\end{gathered}
$$

\subsubsection{Bending and Shear Displacement Sequences:}

Substituting Eq. (20b) into Eq. (18b) and Eq. (18c) leads to the following bending and shear recurrent sequences:

For $\mathrm{k}=0,1,2,3, \ldots$

$$
\mathrm{s}_{\mathrm{k}+4}=\lambda_{\mathrm{k}+2}^{2} \mathrm{k}_{\mathrm{s}} \mathrm{s}_{\mathrm{k}+2}+\lambda_{\mathrm{k}}^{4} \mathrm{E}_{\mathrm{s}} \mathrm{q}_{\mathrm{k}}
$$




$$
\mathrm{b}_{\mathrm{k}+4}=\lambda_{\mathrm{k}+2}^{2} \mathrm{k}_{\mathrm{b}} \mathrm{s}_{\mathrm{k}+2}+\lambda_{\mathrm{k}}^{4} \mathrm{E}_{\mathrm{b}} \mathrm{q}_{\mathrm{k}}
$$

Where

$$
\begin{gathered}
\mathrm{k}_{\mathrm{s}}=\mathrm{C}_{1} \mathrm{k}_{\mathrm{u}}+\mathrm{C}_{2}, \mathrm{E}_{\mathrm{s}}=\mathrm{C}_{1} \mathrm{E}_{\mathrm{u}}+\mathrm{C}_{3} \\
\mathrm{k}_{\mathrm{b}}=\mathrm{B}_{1} \mathrm{k}_{\mathrm{u}}+\mathrm{B}_{2}, \mathrm{E}_{\mathrm{b}}=\mathrm{B}_{1} \mathrm{E}_{\mathrm{u}}+\mathrm{B}_{3}
\end{gathered}
$$

\subsection{Coefficients of the Displacement Series}

\subsubsection{Shear Displacement Series Coefficients}

From the Eq. (22a), we obtain the coefficients of the shear displacement series as follows:

For $\mathrm{i}=2,3,4, \ldots$

$$
\begin{gathered}
\mathrm{s}_{2 \mathrm{i}}=\mathrm{k}_{2 \mathrm{i}}^{\mathrm{s}} \mathrm{s}_{2}+\mathrm{F}_{2 \mathrm{i}}^{\mathrm{s}} \\
\mathrm{s}_{2 \mathrm{i}+1}=\mathrm{k}_{2 \mathrm{i}+1}^{\mathrm{s}} \mathrm{s}_{3}+\mathrm{F}_{2 \mathrm{i}+1}^{\mathrm{s}}
\end{gathered}
$$

In which

$$
\begin{gathered}
\mathrm{k}_{2 \mathrm{i}}^{\mathrm{s}}=\frac{2 !}{\mathrm{k}_{\mathrm{s}}} \frac{\mathrm{k}_{\mathrm{s}}^{\mathrm{i}}}{(2 \mathrm{i}) !} \\
\mathrm{F}_{2 \mathrm{i}}^{\mathrm{s}}=\frac{\mathrm{E}_{\mathrm{S}}}{(2 \mathrm{i}) !} \sum_{\mathrm{n}=0}^{\mathrm{i}-2}(2 \mathrm{n}) ! \mathrm{q}_{2 \mathrm{n}} \mathrm{k}_{\mathrm{s}}^{\mathrm{i}-\mathrm{n}-2} \\
\mathrm{k}_{2 \mathrm{i}+1}^{\mathrm{s}}=\frac{3 !}{\left(\mathrm{k}_{\mathrm{s}}\right)^{3 / 2}} \frac{\mathrm{k}_{\mathrm{s}}^{\mathrm{i}+\frac{1}{2}}}{(2 \mathrm{i}+1) !} \\
\mathrm{F}_{2 \mathrm{i}+1}^{\mathrm{s}}=\frac{\mathrm{E}_{\mathrm{S}}}{(2 \mathrm{i}+1) !} \sum_{\mathrm{n}=0}^{\mathrm{i}-2}(2 \mathrm{n}+1) ! \mathrm{q}_{2 \mathrm{n}+1}\left(k_{s}\right)^{\mathrm{i}-\mathrm{n}-2}
\end{gathered}
$$

\subsubsection{Bending Displacement Series Coefficients}

From Eq. (22b) we obtain the coefficients of the bending displacement series as follows:

For $\mathrm{i}=2,3,4, \ldots$

$$
\begin{array}{r}
\mathrm{b}_{2 \mathrm{i}}=\mathrm{k}_{2 \mathrm{i}}^{\mathrm{b}} \mathrm{s}_{2}+\mathrm{F}_{2 \mathrm{i}}^{\mathrm{b}} \\
\mathrm{b}_{2 \mathrm{i}+1}=\mathrm{k}_{2 \mathrm{i}+1}^{\mathrm{b}} \mathrm{s}_{3}+\mathrm{F}_{2 \mathrm{i}+1}^{\mathrm{b}} \\
\mathrm{k}_{2 \mathrm{i}}^{\mathrm{b}}=\frac{2 ! \mathrm{k}_{\mathrm{b}}}{\mathrm{k}_{\mathrm{s}}^{2}} \frac{\mathrm{k}_{\mathrm{s}}^{\mathrm{i}}}{(2 \mathrm{i}) !} \\
\mathrm{k}_{2 \mathrm{i}+1}^{\mathrm{b}}=\frac{3 ! \mathrm{k}_{\mathrm{b}}}{\left(\mathrm{k}_{\mathrm{s}}\right)^{5 / 2}} \frac{\mathrm{k}_{\mathrm{s}}^{\mathrm{i}+\frac{1}{2}}}{(2 \mathrm{i}+1) !}
\end{array}
$$

For $\mathrm{i}=2$

$$
\begin{gathered}
\mathrm{F}_{4}^{\mathrm{b}}=\frac{1 !}{4 !} \mathrm{q}_{0} \mathrm{E}_{\mathrm{b}} \\
\mathrm{F}_{5}^{\mathrm{b}}=\frac{1 !}{5 !} \mathrm{q}_{1} \mathrm{E}_{\mathrm{b}}
\end{gathered}
$$

For $\mathrm{i}=3,4,5, \ldots$

$$
\begin{gathered}
\mathrm{F}_{2 \mathrm{i}}^{\mathrm{b}}=\mathrm{k}_{\mathrm{b}} \mathrm{E}_{\mathrm{s}} \frac{1}{(2 \mathrm{i}) !} \sum_{\mathrm{n}=0}^{\mathrm{i}-3}(2 \mathrm{n}) ! \mathrm{q}_{2 \mathrm{n}} \mathrm{k}_{\mathrm{s}}^{\mathrm{i}-\mathrm{n}-3}+\mathrm{E}_{\mathrm{b}} \frac{(2 \mathrm{i}-4) !}{(2 \mathrm{i}) !} \mathrm{q}_{2 \mathrm{i}-4} \\
\mathrm{~F}_{2 \mathrm{i}+1}^{\mathrm{b}}=\mathrm{k}_{\mathrm{b}} \mathrm{E}_{\mathrm{s}} \frac{1}{(2 \mathrm{i}+1) !} \sum_{\mathrm{n}=0}^{\mathrm{i}-3}(2 \mathrm{n}+1) ! \mathrm{q}_{2 \mathrm{n}+1} \mathrm{k}_{\mathrm{s}}^{\mathrm{i}-\mathrm{n}-3}+ \\
\mathrm{E}_{\mathrm{b}} \frac{(2 \mathrm{i}-3) !}{(2 \mathrm{i}+1) !} \mathrm{q}_{2 \mathrm{i}-3}
\end{gathered}
$$

\subsubsection{Axial Displacement Series Coefficients}

From Eq. (20b) we obtain the coefficients of the bending displacement series as follows
For $\mathrm{i}=2,3,4, \ldots$

$$
\begin{aligned}
& \mathrm{u}_{2 \mathrm{i}}=\mathrm{k}_{2 \mathrm{i}}^{\mathrm{u}} \mathrm{s}_{3}+\mathrm{F}_{2 \mathrm{i}}^{\mathrm{u}} \\
& \mathrm{k}_{2 \mathrm{i}}^{\mathrm{u}}=\frac{3 ! \mathrm{k}_{\mathrm{u}}}{\mathrm{k}_{\mathrm{s}}^{2}} \frac{\mathrm{k}_{\mathrm{s}}^{\mathrm{i}}}{(2 \mathrm{j}) !}
\end{aligned}
$$

For $\mathrm{i}=1,2,3, \ldots$

$$
\begin{gathered}
\mathrm{u}_{2 \mathrm{i}+1}=\mathrm{k}_{2 \mathrm{i}+1}^{\mathrm{u}} \mathrm{s}_{2}+\mathrm{F}_{2 \mathrm{i}+1}^{\mathrm{u}} \\
k_{2 i+1}^{u}=\frac{2 ! k_{u}}{\left(k_{S}\right)^{3 / 2}} \frac{k_{s}^{i+\frac{1}{2}}}{(2 i+1) !} \\
\mathrm{F}_{4}^{\mathrm{u}}=\frac{1 !}{4 !} \mathrm{q}_{1} \mathrm{E}_{\mathrm{u}}, \\
F_{3}^{u}=\frac{1 !}{3 !} q_{0} E_{u}
\end{gathered}
$$

For $\mathrm{i}=3,4,5, \ldots$

$$
\begin{gathered}
\mathrm{F}_{2 \mathrm{i}}^{\mathrm{u}}=\mathrm{k}_{\mathrm{u}} \mathrm{E}_{\mathrm{s}} \frac{1}{(2 \mathrm{i}) !} \sum_{\mathrm{n}=0}^{\mathrm{i}-3}(2 \mathrm{n}+1) ! \mathrm{q}_{2 \mathrm{n}+1} \mathrm{k}_{\mathrm{s}}^{\mathrm{i}-3-\mathrm{n}}+ \\
\mathrm{E}_{\mathrm{u}} \frac{(2 \mathrm{i}-3) !}{(2 \mathrm{i}) !} \mathrm{q}_{2 \mathrm{i}-3}
\end{gathered}
$$

For $\mathrm{i}=2,3,4,5, \ldots$

$\mathrm{k}_{\mathrm{u}} \mathrm{E}_{\mathrm{s}} \frac{1}{(2 \mathrm{i}+1) !} \sum_{\mathrm{n}=0}^{\mathrm{i}-2}(2 \mathrm{n}) ! \mathrm{q}_{2 \mathrm{n}} \mathrm{k}_{\mathrm{s}}^{\mathrm{i}-2-\mathrm{n}}+\mathrm{E}_{\mathrm{u}} \frac{(2 \mathrm{i}-2) !}{(2 \mathrm{i}+1) !} \mathrm{q}_{2 \mathrm{i}-2} \mathrm{~F}_{2 \mathrm{i}+1}^{\mathrm{u}}=$

The previous Eq. (24), Eq. (25) and Eq. (26) leads to the following polynomial forms:

$$
\begin{array}{rr}
\mathrm{w}_{\mathrm{s}}(\mathrm{x})=\mathrm{s}_{0}+\mathrm{s}_{1} \mathrm{x}+\mathrm{s}_{2} \mathrm{x}^{2}+\mathrm{s}_{3} \mathrm{x}^{3}+\sum_{\mathrm{i}=2}^{\infty}\left(\mathrm{s}_{2 \mathrm{i}} \mathrm{x}^{2 \mathrm{i}}\right)+ \\
\sum_{\mathrm{i}=2}^{\infty}\left(\mathrm{s}_{2 \mathrm{i}+1} \mathrm{x}^{2 \mathrm{i}+1}\right) & (27 \mathrm{a}) \\
\mathrm{w}_{\mathrm{b}}(\mathrm{x})=\mathrm{b}_{0}+\mathrm{b}_{1} \mathrm{x}+\mathrm{b}_{2} \mathrm{x}^{2}+\mathrm{b}_{3} \mathrm{x}^{3}+\sum_{\mathrm{i}=2}^{\infty}\left(\mathrm{b}_{2 \mathrm{i}} \mathrm{x}^{2 \mathrm{i}}\right)+ \\
\sum_{\mathrm{i}=2}^{\infty}\left(\mathrm{b}_{2 \mathrm{i}+1} \mathrm{x}^{2 \mathrm{i}+1}\right) & (27 \mathrm{~b}) \\
\mathrm{u}_{0}+\mathrm{u}_{1} \mathrm{x}+\underset{\mathrm{u}(\mathrm{x})=}{\left(3 \mathrm{~A}_{1} \mathrm{~b}_{3}+3 \mathrm{~A}_{2} \mathrm{~s}_{3}\right) \mathrm{x}^{2}+\sum_{\mathrm{i}=2}^{\infty}\left(\mathrm{u}_{2 \mathrm{i}} \mathrm{x}^{2 \mathrm{i}}\right)+} \\
\sum_{\mathrm{i}=1}^{\infty}\left(\mathrm{u}_{2 \mathrm{i}+1} \mathrm{x}^{2 \mathrm{i}+1}\right) & (27 \mathrm{c})
\end{array}
$$

where $\mathrm{u}_{0}, \mathrm{u}_{1}, \mathrm{~b}_{0}, \mathrm{~b}_{1}, \mathrm{~b}_{2}, \mathrm{~b}_{3}, \mathrm{~s}_{0}, \mathrm{~s}_{1}, \mathrm{~s}_{2}$ and $\mathrm{s}_{3}$ are determined once the boundary conditions(BCs) are defined

\subsection{Displacement Series Form}

\subsubsection{Shear Displacement Series}

From Eqs. (24c) and Eq.(24e) we obtain by using the Taylor's series expansion:

$$
\sum_{\mathrm{i}=2}^{+\infty} \mathrm{k}_{2 \mathrm{i}}^{\mathrm{s}} \cdot \mathrm{x}^{2 \mathrm{i}}=\frac{2 !}{\mathrm{k}_{\mathrm{s}}}\left(\cosh \left(\sqrt{\mathrm{k}_{\mathrm{s}} \mathrm{x}}\right)-1\right)-\mathrm{x}^{2}
$$

$\sum_{i=2}^{+\infty} k_{2 j+1}^{s} \cdot x^{2 j+1}=\frac{3 !}{\left(k_{s}\right)^{3 / 2}}\left(\sinh \left(\sqrt{k_{s}} x\right)-\sqrt{k_{s}} x\right)-x^{3}$

Hence [19]

$$
\begin{array}{r}
\mathrm{w}_{\mathrm{s}}(\mathrm{x})=\mathrm{s}_{0}+\mathrm{s}_{1} \mathrm{x}+\frac{2}{\mathrm{k}_{\mathrm{s}}}\left(\cosh \left(\sqrt{\mathrm{k}_{\mathrm{s}} \mathrm{x}}\right)-1\right) \mathrm{s}_{2}+ \\
\frac{6}{\left(\mathrm{k}_{\mathrm{s}}\right)^{3 / 2}}\left(\sinh \left(\sqrt{\mathrm{k}_{\mathrm{s}} \mathrm{x}}\right)-\sqrt{\mathrm{k}_{\mathrm{s}} \mathrm{x}}\right) \mathrm{s}_{3}+\mathrm{r}_{\mathrm{s}}(\mathrm{x})
\end{array}
$$

Where

$$
r_{s}(x)=\sum_{2}^{\infty} F_{2 i}^{s} x^{2 i}+\sum_{2}^{\infty} F_{2 i+1}^{s} x^{2 i+1}
$$


$r_{s}(x)$ is expressed once the load form is defined 5.4.2. Bending Displacement Solution

From Eq. (25c) we obtain by using the Taylor's series expansion:

$$
\begin{gathered}
\sum_{\mathrm{i}=2}^{+\infty} \mathrm{k}_{2 \mathrm{i}}^{\mathrm{b}} \cdot \mathrm{x}^{2 \mathrm{i}}=\frac{2 ! \mathrm{k}_{\mathrm{b}}}{\mathrm{k}_{\mathrm{s}}^{2}}\left(\cosh \left(\sqrt{k_{s}} x\right)-1\right)-\frac{\mathrm{k}_{\mathrm{b}}}{\mathrm{k}_{\mathrm{s}}} \mathrm{x}^{2} \\
\sum_{\mathrm{i}=2}^{+\infty} \mathrm{k}_{2 \mathrm{i}+1}^{\mathrm{b}} \cdot \mathrm{x}^{2 \mathrm{i}+1}=\frac{3 ! \mathrm{k}_{\mathrm{b}}}{\left(\mathrm{k}_{\mathrm{s}}\right)^{5} / 2}\left(\sinh \left(\sqrt{k_{s}} x\right)-\sqrt{\mathrm{k}_{\mathrm{s}}} \mathrm{x}\right)-\frac{\mathrm{k}_{\mathrm{b}}}{\mathrm{k}_{\mathrm{s}}} \mathrm{x}^{3}
\end{gathered}
$$

Hence, the final general form of the bending displacement expression is given by [19]:

$$
\begin{array}{r}
\mathrm{w}_{\mathrm{b}}(\mathrm{x})= \\
\mathrm{b}_{0}+\mathrm{b}_{1} \mathrm{x}+\mathrm{b}_{2} \mathrm{x}^{2}+\mathrm{b}_{3} \mathrm{x}^{3}+\frac{\mathrm{k}_{\mathrm{b}}}{\mathrm{k}_{\mathrm{s}}{ }^{2}}\left(2\left[\cosh \left(\sqrt{\mathrm{k}_{\mathrm{s}} \mathrm{x}}\right)-1\right]-\right. \\
\left.\mathrm{k}_{\mathrm{s}} \mathrm{x}^{2}\right) \mathrm{s}_{2}+\frac{\mathrm{k}_{\mathrm{b}}}{\mathrm{k}_{\mathrm{s}}{ }^{5} / 2}\left\{6\left(\sinh \left(\sqrt{\mathrm{k}_{\mathrm{s}}} \mathrm{x}\right)-\sqrt{\mathrm{k}_{\mathrm{s}} \mathrm{x}}\right)-\mathrm{k}_{\mathrm{s}}{ }^{3 / 2} \mathrm{x}^{3}\right\} \mathrm{s}_{3}+ \\
\mathrm{r}_{\mathrm{b}}(\mathrm{x})
\end{array}
$$

In which

$$
r_{b}(x)=\sum_{2}^{\infty} F_{2 i}^{b} x^{2 i}+\sum_{2}^{\infty} F_{2 i+1}^{b} x^{2 i+1}
$$

$r_{b}(x)$ is expressed once the load form is defined.

\subsubsection{Axial Displacement Solution}

From Eqs. (26b) and Eq.(26d) we obtain using the Taylor's series expansion:

$$
\begin{gathered}
\sum_{\mathrm{i}=2}^{+\infty} \mathrm{k}_{2 \mathrm{i}}^{\mathrm{u}} \cdot \mathrm{x}^{2 \mathrm{i}}=\frac{3 ! \mathrm{k}_{\mathrm{u}}}{\mathrm{k}_{\mathrm{s}}{ }^{2}}\left(\cosh \left(\sqrt{\mathrm{k}_{\mathrm{s}}} \mathrm{x}\right)-1\right)-3 \frac{\mathrm{k}_{\mathrm{u}}}{\mathrm{k}_{\mathrm{s}}} \mathrm{x}^{2} \\
\sum_{\mathrm{i}=1}^{+\infty} \mathrm{k}_{2 \mathrm{i}+1}^{\mathrm{u}} \cdot \mathrm{x}^{2 \mathrm{i}+1}=\frac{2 ! \mathrm{k}_{\mathrm{u}}}{\mathrm{k}_{\mathrm{s}} / 2} \sinh \left(\sqrt{\mathrm{k}_{\mathrm{s}} \mathrm{x}}\right)-2 \frac{\mathrm{k}_{\mathrm{u}}}{\mathrm{k}_{\mathrm{s}}} \mathrm{x}
\end{gathered}
$$

Hence, the final general form of the axial displacement expression is given by[19]:

$$
\begin{array}{r}
u(x)=u_{0}+u_{1} x+\frac{2 k_{u}}{k_{s}^{3} / 2}\left(\sinh \left(\sqrt{k_{s}} x\right)-\sqrt{k_{s}} x\right) s_{2}+ \\
\frac{6 k_{u}}{k_{s}{ }^{2}}\left(\cosh \left(\sqrt{k_{s} x}\right)-1\right) s_{3}+3\left(A_{2}-\frac{k_{u}}{k_{s}}\right) x^{2} s_{3}+ \\
3 A_{1} x^{2} b_{3}+r_{u}(x)
\end{array}
$$

Where

$$
\mathrm{r}_{\mathrm{u}}(\mathrm{x})=\sum_{2}^{\infty} \mathrm{F}_{2 \mathrm{i}}^{\mathrm{u}} \mathrm{x}^{2 \mathrm{i}}+\sum_{1}^{\infty} \mathrm{F}_{2 \mathrm{i}+1}^{\mathrm{u}} \mathrm{x}^{2 \mathrm{i}+1}
$$

$r_{u}(x)$ is expressed once the load form is defined.

\section{Load Form Series}

The final exact forms of $r_{u}(x)$ given by Eq.(30d), $r_{b}(x)$ given by Eq.(29d) and $r_{s}(x)$ given by Eq.(28d) are to be expressed for each external load. In this section we develop the uniformly distributed load.

For uniform load we have

$$
\mathrm{q}(\mathrm{x})=\mathrm{q}_{\mathrm{o}}
$$

By applying DTM we obtain

$$
\mathrm{q}_{\mathrm{k}}=\mathrm{q}_{\mathrm{o}} \text { if } \mathrm{k}=0, \mathrm{q}_{\mathrm{k}}=0 \text { otherwise }
$$

Substituting Eq. (31b) into Eq. (24d), Eq. (24f), Eq. (25d), Eq. (25e), Eq. (25f), Eq. (26e), Eq. (26f) and Eq. $(26 \mathrm{~g})$ yields [19]

$$
\begin{gathered}
r_{s}(x)=\frac{E_{s} q_{0}}{k_{s}{ }^{2}}\left(\cosh \left(\sqrt{k_{s}} x\right)-1-\frac{k_{s} x^{2}}{2 !}\right) \\
r_{b}(x)=\frac{E_{s} k_{b} q_{0}}{k_{s}{ }^{3}}\left(\cosh \left(\sqrt{k_{s} x}\right)-1-\frac{k_{s} x^{2}}{2 !}\right)+\frac{E_{s} q_{0} x^{4}}{4 !}\left(\frac{E_{b}}{E_{s}}-\right. \\
\left.\frac{k_{b}}{k_{s}}\right) \\
r_{u}(x)=\frac{E_{s} k_{u} q_{0}}{k_{s}^{5} / 2}\left(\sinh \left(\sqrt{k_{s}} x\right)-\sqrt{k_{s} x}\right)+\frac{E_{s} q_{0} x^{3}}{3 !}\left(\frac{E_{u}}{E_{s}}-\frac{k_{u}}{k_{s}}\right)
\end{gathered}
$$

\section{Boundary Conditions and Relevant Exact Analytical Solution: Clamped-Clamped Beam}

In this section, the exact analytical solution is given for the clamped-clamped FG beam bending under uniform load fig. 2:

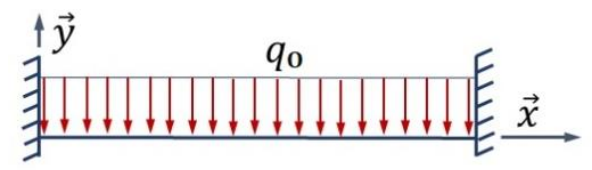

Figure 2. Clamped - Clamped FG beam under uniform load In this case the system to solve is the one given by the boundary conditions as follows:

$$
\begin{gathered}
\mathrm{u}(\mathrm{x}=0)=0 \text { yields } \mathrm{u}_{0}=0 \\
\mathrm{w}_{\mathrm{b}}(\mathrm{x}=0)=0 \text { yields } \mathrm{b}_{0}=0 \\
\mathrm{w}_{\mathrm{s}}(\mathrm{x}=0)=0 \text { yields } \mathrm{s}_{0}=0 \\
\frac{\mathrm{dw_{ \textrm {s } }}}{\mathrm{dx}}(\mathrm{x}=0)=0, \\
\frac{d w_{\mathrm{b}}}{\mathrm{dx}}(\mathrm{x}=0)=0 \\
\frac{d \mathrm{w}_{\mathrm{s}}}{\mathrm{dx}}(\mathrm{x}=\mathrm{L})=0, \frac{\mathrm{d} \mathrm{w}_{\mathrm{b}}}{\mathrm{dx}}(\mathrm{x}=\mathrm{L})=0, \\
\mathrm{w}_{\mathrm{s}}(\mathrm{x}=\mathrm{L})=0, \mathrm{w}_{\mathrm{b}}(\mathrm{x}=\mathrm{L})=0, \mathrm{u}(\mathrm{x}=\mathrm{L})=0
\end{gathered}
$$

Substituting the subsequent result of solving the system given by Eqs. (33) into Eq. (28c), Eq.(29c) and Eq.(30c) leads to the following final expressions:

\subsection{The Exact Analytical Solution}

7.1.1. Shear Displacement

$$
\begin{aligned}
\mathrm{W}_{\mathrm{S}}\left(\mathrm{x}^{*}\right) & =\alpha \frac{\mathrm{E}_{\mathrm{S}} \mathrm{q}_{0}}{2 \mathrm{k}_{\mathrm{s}}{ }^{2}}\left[\frac{\sinh \left(\alpha\left(1-\mathrm{x}^{*}\right)\right)+\sinh \left(\alpha \mathrm{x}^{*}\right)-\sinh \alpha}{\cosh \alpha-1}+\right. \\
& \left.\alpha \mathrm{x}^{*}\left(1-\mathrm{x}^{*}\right)\right]
\end{aligned}
$$

7.1.2. Bending displacement

$$
\begin{aligned}
& \mathrm{w}_{\mathrm{b}}\left(\mathrm{x}^{*}\right)= \\
& \frac{E_{s} k_{b} q_{0}}{2 k_{s}{ }^{3}} \alpha\left[\alpha x^{*}\left(1-x^{*}\right)+\frac{\sinh \left[\alpha\left(1-x^{*}\right)\right]+\sinh \left(\alpha x^{*}\right)-\sinh \alpha}{\cosh \alpha-1}\right]+ \\
& \frac{E_{s} q_{0}}{24 k_{s}^{2}} \alpha^{4}\left(\frac{E_{b}}{E_{s}}-\frac{k_{b}}{k_{s}}\right)\left(1-x^{*}\right)^{2} x^{* 2}
\end{aligned}
$$


7.1.3. Axial Displacement

$$
\begin{aligned}
& \mathrm{u}\left(\mathrm{x}^{*}\right)=\alpha\left(\frac{\mathrm{E}_{\mathrm{s}} \mathrm{k}_{\mathrm{u}} \mathrm{q}_{0}}{\mathrm{k}_{\mathrm{s}}^{5} / 2}\right)\left\{\frac{1}{2}-\mathrm{x}^{*}+\frac{\cosh \left(\alpha \mathrm{x}^{*}\right)-\cosh \left[\alpha\left(1-\mathrm{x}^{*}\right)\right]}{2(\cosh \alpha-1)}\right\}+ \\
& \frac{1}{6} \frac{\mathrm{E}_{\mathrm{s}} \mathrm{q}_{0}}{\mathrm{k}_{\mathrm{s}}{ }^{3 / 2}} \alpha^{3}\left(\frac{\mathrm{E}_{\mathrm{u}}}{\mathrm{E}_{\mathrm{s}}}-\frac{\mathrm{k}_{\mathrm{u}}}{\mathrm{k}_{\mathrm{s}}}\right) \mathrm{x}^{*}\left(1-\mathrm{x}^{*}\right)\left[\frac{1}{2}-\mathrm{x}^{*}\right]
\end{aligned}
$$

These functions are rewritten for Homogeneous and is otropic beam as follows (see the appendix B)

$$
\begin{gathered}
w_{b}\left(\mathrm{x}=\frac{\mathrm{L}}{2}\right)=\frac{1}{384} \frac{\mathrm{q}_{0} \mathrm{~L}^{4}}{\mathrm{E}_{\mathrm{c}, \mathrm{m}} \mathrm{I}}-\frac{3}{80} \frac{\mathrm{q}_{0} \mathrm{~L}^{2}}{\mathrm{G}_{\mathrm{c}, \mathrm{m}} \mathrm{S}}\left[1-\frac{\tanh \left(\frac{\alpha}{4}\right)}{\frac{\alpha}{4}}\right] \\
\mathrm{w}_{\mathrm{S}}(L / 2)=\frac{3}{16} \frac{q_{0} L^{2}}{G_{c, m} S}\left[1-\frac{\tanh \left(\frac{\alpha}{4}\right)}{\alpha / 4}\right] \\
\mathrm{u}(\mathrm{x})=0
\end{gathered}
$$

The maximum transverse displacement is, then, given by:

$$
\mathrm{W}=\frac{1}{384} \frac{\mathrm{q}_{0} \mathrm{~L}^{4}}{\mathrm{E}_{\mathrm{c}, \mathrm{m}} \mathrm{I}}+\frac{3}{20} \frac{\mathrm{q}_{0} \mathrm{~L}^{2}}{\mathrm{G}_{\mathrm{c}, \mathrm{m}} \mathrm{S}}\left[1-\frac{\tanh \left(\frac{\alpha}{4}\right)}{\alpha / 4}\right]
$$

In the deflection expression Eq. (36), the first term shows the contribution according to the classical Euler-Bernoulli beam theory and the second term represents the effect of transverse shear deformation. The table 2 indicates numerical values for maximu m trans verse displacement of a FGM composed of Aluminum $(\mathrm{Em}=70 \mathrm{GPa}, \quad v=0.3)$ and Zirconia (Ec=200GPa, $v=0.3)[2]$ (normalized by $\frac{1}{384} \frac{\mathrm{q}_{0} \mathrm{~L}^{4}}{\mathrm{E}_{\mathrm{m}} \mathrm{I}}$ for clamped beam ). It can be observed that the exact analytical results are close to those given by Vo and al [6].

Figs 3 and Fig 4 illustrate the transverse and axial displacements (given by the exact analytical solution) for various values of power law index $\mathrm{n}$ of $\mathrm{FG}$ beam under uniform load and for $(\mathrm{L} / \mathrm{h}=4, \mathrm{~L} / \mathrm{h}=16)$. Those displacements are normalized by $\frac{1}{384} \frac{\mathrm{q}_{0} \mathrm{~L}^{4}}{\mathrm{E}_{\mathrm{m}} \mathrm{I}}$. We can note that the mid-point has no axial displacement.

Fig 5 illustrates the axial normal and shear stresses for various values of power law index $n$ of FG beam under uniform load and for $(\mathrm{L} / \mathrm{h}=4, \mathrm{~L} / \mathrm{h}=20)$. We can notice that the shear stress value does not fluctuate considerably while changing the value of $\mathrm{L} / \mathrm{h}$

Table 2. Nondimensional maximum displacements of FG beams with various values of power law exponent for different boundary conditions for $\mathrm{Em}=70 \mathrm{GPa}, \mathrm{Ec}=200 \mathrm{GPa}$ and $v=0.3$

\begin{tabular}{|c|c|c|c|c|c|c|}
\hline $\mathrm{L} / \mathrm{h}$ & Method & $\mathrm{n}=0$ Full ceramic & $\mathrm{n}=1$ & $\mathrm{n}=5$ & $\mathrm{n}=10$ & Full metal \\
\hline \multirow{2}{*}{4} & Present & 0,60781 & 0,95355 & 1,31843 & 1,43822 & 1,73660 \\
\cline { 2 - 7 } & Vo and al [6] & 0.60773 & 0.94365 & 1.31813 & 1.43793 & 1.73637 \\
\hline \multirow{2}{*}{16} & Present & 0,36683 & 0,58702 & 0,74515 & 0,80609 & 1,04807 \\
\cline { 2 - 7 } & Vo and al [6] & 0.36676 & 0.58667 & 0.74488 & 0.80586 & 1.04789 \\
\hline
\end{tabular}

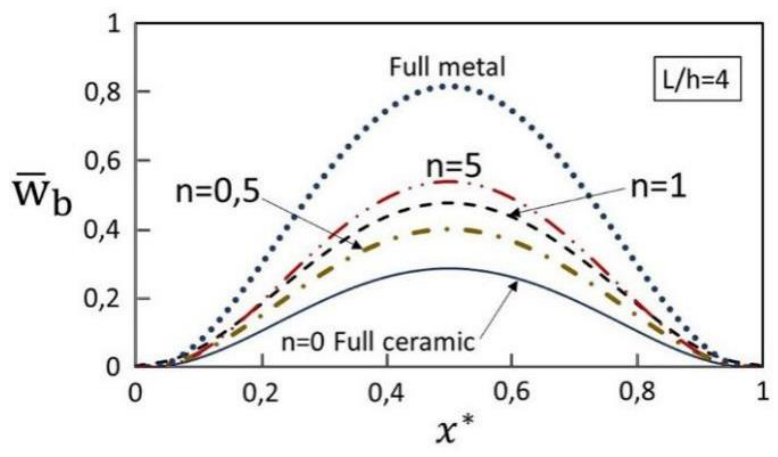

Figure 3a. Nondimensional transverse bending displacement given by the exact analytical solution for clamped-clamped FG beam bending under uniform load 


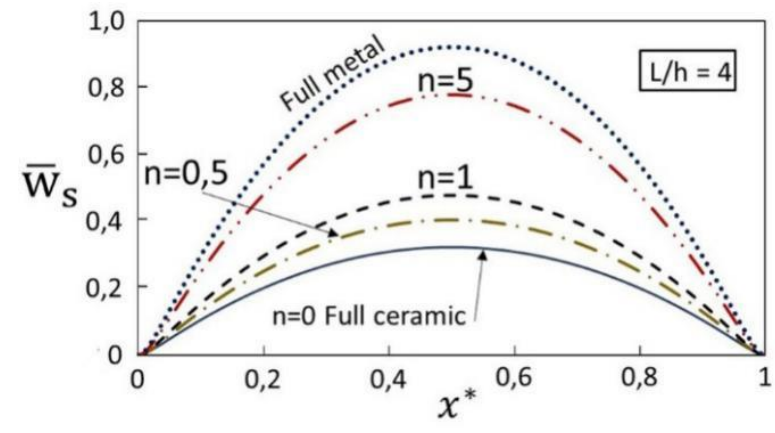

Figure 3b. Nondimensional shear bending displacement given by the exact analytical solution for clamped-clamped FG beam bending under uniform load

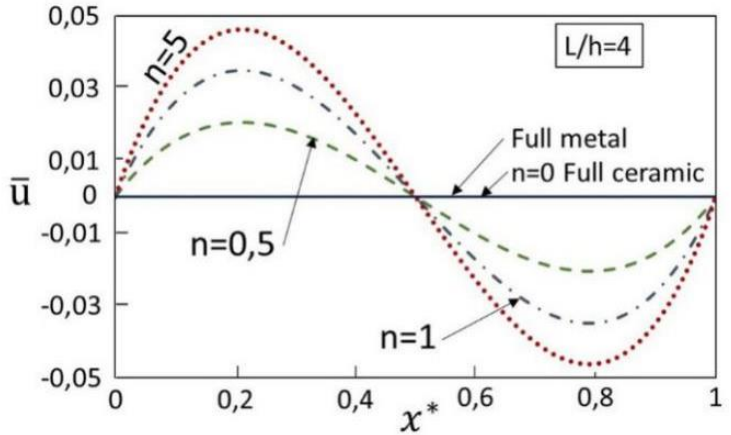

Figure 4. Nondimensional Axial displacement given by the exact analytical solution for clamped-clamped FG beam bending under uniform $\operatorname{load}(\mathrm{L}=4 \mathrm{~h})$
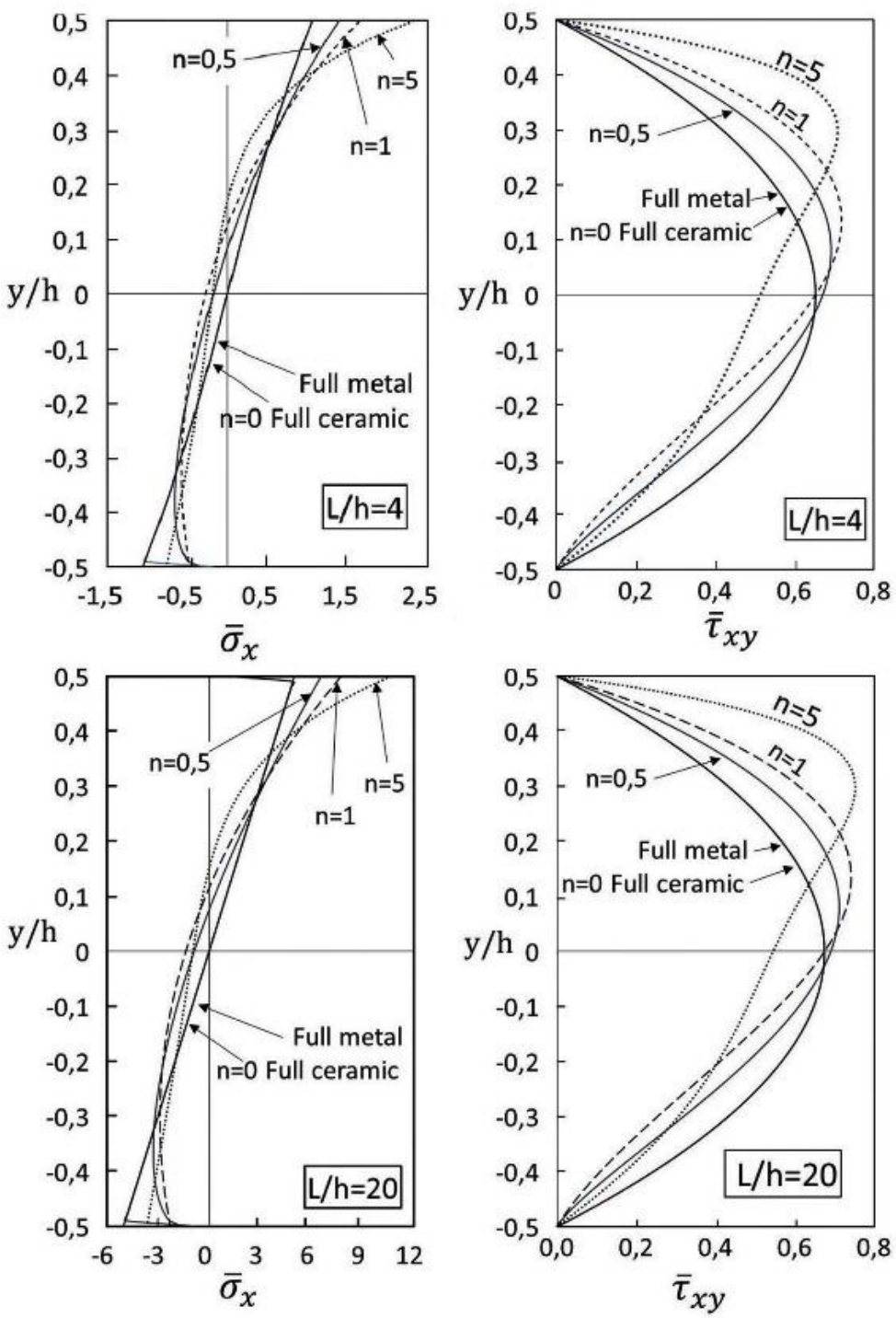

Figure 5. The axial normal and shear stresses for various values of power law index $\mathrm{n}$ of $\mathrm{FG}$ beam under uniform load and for $(\mathrm{L} / \mathrm{h}=4$, $\mathrm{L} / \mathrm{h}=20) . \mathrm{Em}=70 \mathrm{GPa}, \mathrm{Ec}=380 \mathrm{GPa}, v=0.3$ 


\section{Conclusions}

The analytical solution to the static bending problem of FGM beams with HOSDT was presented and applied to the case of a constant cross-section recessed-embedded beam under the action of a UDL. The results obtained clearly show the possibility of analyzing the shear effects as well as taking into account the effect of the different parameters related to the FGM law. The comparis on of the results with those obtained by a numerical resolution confirms the effectiveness of the DTM method. This approach, which is successfully used to solve this type of ODE equations, naturally coupled, can be applied to solve physics or engineering problems governed by the same type of differential equation systems. It should also be noted that numerical solving is, in this case, not necessary.

\section{Appendix A: Coefficients (Used in the Eqs. (12)) for Rectangular Cross Section of the FG Beam}

$$
\begin{gathered}
\mathrm{A}=\int_{-\mathrm{h} / 2}^{\mathrm{h} / 2} \mathrm{E}(\mathrm{y}) \mathrm{bdy}=\mathrm{bh} \frac{\mathrm{nE}_{\mathrm{m}}+\mathrm{E}_{\mathrm{c}}}{\mathrm{n}+1} \\
B=\int_{-\mathrm{h} / 2}^{\mathrm{h} / 2} \mathrm{E}(\mathrm{y}) \mathrm{ybdy}=\mathrm{bh}\left(\mathrm{E}_{\mathrm{c}}-\mathrm{E}_{\mathrm{m}}\right) \frac{\mathrm{hn}}{2(\mathrm{n}+1)(\mathrm{n}+2)} \\
\mathrm{D}=\int_{-\frac{\mathrm{h}}{2}}^{\frac{\mathrm{h}}{2}} \mathrm{E}(\mathrm{y}) \mathrm{y}^{2} \mathrm{bdy}=\mathrm{E}_{\mathrm{m}} \frac{\mathrm{bh}^{3}}{12}+\left(\mathrm{E}_{\mathrm{c}}-\mathrm{E}_{\mathrm{m}}\right) \mathrm{bh}^{3}\left(\frac{1}{4(\mathrm{n}+1)}-\right. \\
\left.\frac{1}{\mathrm{n}+2}+\frac{1}{\mathrm{n}+3}\right)
\end{gathered}
$$

For TBT based on Reddy [16]

$$
f(y)=\frac{4 y^{3}}{3 h^{2}}, g(y)=1-\frac{4 y^{2}}{h^{2}}
$$

$$
B_{s}=\int_{-h / 2}^{h / 2} E(y) f(y) b d y=\frac{4}{3} b h^{2}\left(E_{c}-E_{m}\right)\left(-\frac{1}{8(n+1)}+\right.
$$

$$
\left.\frac{3}{4(n+2)}-\frac{3}{2(n+3)}+\frac{1}{n+4}\right)
$$

$$
\begin{array}{r}
D_{s}=\int_{-h / 2}^{h / 2} E(y) y f(y) b d y= \\
E_{m} \frac{b^{3}}{60}+\left(E_{c}-E_{m}\right) \frac{4 b h^{3}}{3}\left(\frac{1}{16(n+1)}-\frac{1}{2(n+2)}+\frac{3}{2(n+3)}-\right. \\
\left.\frac{2}{n+4}+\frac{1}{n+5}\right) \quad(A 6) \\
H_{s}=\int_{-h / 2}^{h / 2} E(y) f(y)^{2} b d y= \\
E_{m} \frac{b h^{3}}{252}+\left(E_{c}-E_{m}\right) \frac{16 b h^{3}}{9}\left(\frac{1}{64(n+1)}-\frac{3}{16(n+2)}+\frac{15}{16(n+3)}-\right. \\
\left.\frac{5}{2(n+4)}+\frac{15}{4(n+5)}-\frac{3}{n+6}+\frac{1}{n+7}\right) \quad(A 7) \\
A_{m} \frac{8 b h}{15}+\left(G_{c}-G_{m}^{\frac{h}{2}} g(y)^{2} G(y) b d y=\right. \\
\frac{h}{2} g b h\left(\frac{1}{n+3}-\frac{2}{n+4}+\frac{1}{n+5}\right)
\end{array}
$$

\section{Appendix B - Constants Used in This Work}

$$
\Delta=\mathrm{DH}_{\mathrm{s}}-\mathrm{D}_{\mathrm{s}}^{2}
$$

$$
\begin{gathered}
\mathrm{A}_{1}=\frac{\mathrm{B}}{\mathrm{A}}, \mathrm{A}_{2}=\frac{\mathrm{B}_{\mathrm{s}}}{\mathrm{A}} \\
\left(\mathrm{B.} \mathrm{2)} \mathrm{B}_{1}=\frac{\mathrm{BH}_{\mathrm{s}}-\mathrm{B}_{\mathrm{s}} \mathrm{D}_{\mathrm{s}}}{\Delta}, \mathrm{B}_{2}=-\frac{\mathrm{A}_{\mathrm{s}} \mathrm{D}_{\mathrm{s}}}{\Delta},\right. \\
\mathrm{B}_{3}=\frac{\mathrm{H}_{\mathrm{s}}-\mathrm{D}_{\mathrm{s}}}{\Delta} \\
\mathrm{C}_{1}=\frac{\mathrm{B}_{\mathrm{s}} \mathrm{D}-\mathrm{BD}_{\mathrm{s}}}{\Delta}, \\
\mathrm{C}_{2}=\frac{\mathrm{A}_{\mathrm{s}} \mathrm{D}}{\Delta}, \mathrm{C}_{3}=\frac{\mathrm{D}-\mathrm{D}_{\mathrm{s}}}{\Delta}
\end{gathered}
$$

For full ceramic $(n=0)$ or full metallic $(n \rightarrow \infty)$ and according to the appendix A, we obtain.

$$
\begin{gathered}
\mathrm{A}_{1}=0, \mathrm{~A}_{2}=0 \\
\mathrm{~B}_{1}=0, \\
\mathrm{~B}_{2}=-7 \frac{\mathrm{bh}}{\left(1+v_{\mathrm{c}, \mathrm{m}}\right) \mathrm{I}}, \mathrm{B}_{3}=-\frac{20}{\mathrm{E}_{\mathrm{c}, \mathrm{m} I}} \\
\mathrm{C}_{1}=0, \mathrm{C}_{2}=35 \frac{\mathrm{bh}}{\left(1+v_{\mathrm{c}, \mathrm{m}}\right) \mathrm{I}}, \mathrm{C}_{3}=\frac{105}{\mathrm{E}_{\mathrm{c}, \mathrm{m} I}} \\
\mathrm{k}_{\mathrm{s}}=\mathrm{C}_{2}=35 \frac{\mathrm{bh}}{\left(1+v_{\mathrm{c}, \mathrm{m}}\right) \mathrm{I}}, \mathrm{E}_{\mathrm{s}}=\mathrm{C}_{3}=\frac{105}{\mathrm{E}_{\mathrm{c}, \mathrm{m}} \mathrm{I}}, \\
\mathrm{k}_{\mathrm{b}}=0, \mathrm{~B}_{2}=-7 \frac{\mathrm{bh}}{\left(1+\mathrm{v}_{\mathrm{c}, \mathrm{m}}\right) \mathrm{I}}, \\
\mathrm{E}_{\mathrm{b}}=\mathrm{B}_{3}=-\frac{20}{\mathrm{E}_{\mathrm{c}, \mathrm{m}} \mathrm{I}} \\
\alpha=2 \frac{\mathrm{L}}{\mathrm{h}} \sqrt{\frac{105}{\left(1+v_{\mathrm{c}, \mathrm{m}}\right)}}
\end{gathered}
$$

\section{REFERENCES}

[1] Kadoli R, Akhtar K, Ganesan N. Static analysis of functionally graded beams using higher order shear deformation theory. Appl Math Model 2008; 32(12):25092525 https://doi.org/10.1016/j.apm.2007.09.015

[2] Simsek M. Static analysis of a functionally graded beam under a uniformly distributed load by Ritz method. Int J Eng Appl Sci 2009; 1(3):1-11

[3] Giunta G, Belouettar S, Carrera E. Analy sis of FGM beams by means of classical and advanced theories. Mech Adv Mater Struct 2010; 17(8):622-35.

[4] http://dx.doi.org/10.1080/15376494.2010.518930

[5] Zhong Z, Yu T. Analytical solution of a cantilever functionally graded beam. Compos Sci Technol 2007; 67(3-4):481-

488https://doi.org/10.1016/j.compscitech.2006.08.023

[6] Li XF, Wang BL, Han JC. A higher-order theory for static and dy namic analyses of functionally graded beams. Arch Appl Mech 2010; 80(10):1197-1212. https://doi.org/10.10 07/s00419-010-0435-6

[7] Vo, Thuc \& Thai, Tai \& Nguyen, Trung-Kien \& Inam, 
Fawad. Static and vibration analy sis of functionally graded beams using refined shear deformation theory. Meccanica 2013; 49: 155-168. https://doi.org/10.1007/s11012-013-97 $80-1$

[8] Chen, C.K. and Ho, S.H. Application of differential transformation to eigenvalue problems, Applied Mathematics and Computation 1996; 79(2-3), 173-188. https://doi.org/10.1016/0096-3003(95)00253-7

[9] Ayaz, F. Applications of differential transform method to differential-algebraic equations, Applied Mathematics and Computation 2004; 152(3), 649-657. https://doi.org/10.101 6/S0096-3003(03)00581-2

[10] Ayaz, F. Solutions of the system of differential equations by differential transform method, Applied Mathematics and Computation 2004; 147(2), 547-567. https://doi.org/10.101 6/S0096-3003(02)00794-4

[11] Arikoglu, A. and Ozkol, I. Solution of boundary value problems for integro differential equations by using differential transform method, Applied Mathematics and Computation 2005; 168(2), 1145-1158. https://doi.org/10.1 016/j.amc.2004.10.009

[12] Arikoglu A. and Ozkol, I. Solution of differential-difference equations by using diffrential transform method, Applied Mathematics and Computation 2006; 181(1), 153-162. https://doi.org/10.1016/j.amc.2006.01.022

[13] Sarp, Ümit \& Evirgen, Firat \& Ikikardes, Sebahattin. Applications of differential transformation method to solve systems of ordinary and partial differential equations. Journal of Balıkesir University Institute of Science and Technology 2018; 20(2). 135-156. https://doi.org/10.25092 /baunfbed.423145

[14] Markworth AJ, Ramesh KS, Parks WP. Modelling studies applied to functionally graded materials. J Mater Sci 1995 ; 30(9):2183-2193. https://doi.org/10.1007/BF01184560

[15] Reddy JN. A simple higher-order theory for laminated composite plates. J Appl Mech 1984; 51(4):745-752. doi:10.1115/1.3167719

[16] C.M. Wang, J.N. Reddy, K.H. Lee. SHEAR DEFORMABLE BEAMS AND PLATES Relationships with Classical Solutions. Elsevier 2000(1): 11-37. E-Book. ISBN 9780080437842, 9780080541150

[17] Reddy JN. Energy principles and variational methods in applied mechanics 2nd. John Wiley \& Sons Inc; 2002. ISBN: 978-0-471-17985-6

[18] Sayyad, Atteshamuddin \& Ghugal, Yuwaraj. Analytical solutions for bending, buckling, and vibration analy ses of exponential functionally graded higher order beams. Asian Journal of Civil En gineering 2018; 19(5): 607-623. https:// doi.org/10.1007/s42107-018-0046-z

[19] Farhatnia, Fatemeh \& Sarami, Mostafa. (2019). Finite Element Approach of Bending and Buckling Analysis of FG Beams Based on Refined Zigzag Theory. Universal Journal of Mechanical En gineering. 7. 147-158. 10.13189/ujme.20 19.070402 .

[20] RAZOUKI, BOUTAHAR \& EL BIKRI, 2019. the exact analytical solution of the bending analysis of thick functionally graded beams with higher order shear deformation theory using differential transform method. International Journal of Mechanical Engineering and Technology (IJMET). Volume:10, Issue:10, Pages:75-840. 